\title{
Purslane supplementation lowers oxidative stress, inflammatory and muscle damage biomarkers after high-intensity intermittent exercise in female runners
}

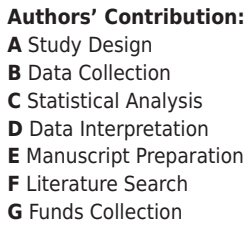

G Funds Collection

\author{
Anis Charkhi Sahl Abad ${ }^{1}$ ABEF, Amine Ghram ${ }^{1,2}$ ACDEF, Rahman Soori ${ }^{1 \text { ABE, }}$ \\ Ali Akbarnejad ${ }^{1}$, Fatemeh Azizi Ghuchan ${ }^{3 \mathrm{AB}}$, Mohammad Mehdi Zare ${ }^{1 \mathrm{AB}}$, \\ Anthony C. Hackney ${ }^{\mathrm{EF}}$, Stephen J. Bailey ${ }^{\mathrm{EF}}$ \\ ${ }^{1}$ Department of Exercise Physiology, Faculty of Physical Education and Sport Sciences, \\ University of Tehran, Tehran, Iran \\ 2 Department of Cardiac Rehabilitation, Tehran Heart Center, \\ Tehran University of Medical Sciences, Tehran, Iran \\ ${ }^{3}$ Department of Exercise Physiology, Bojnourd Branch, Islamic Azad University, Bojnourd, Iran \\ ${ }^{4}$ Department of Exercise \& Sport Science, University of North Carolina, Chapel Hill, NC, USA \\ ${ }^{5}$ School of Sport, Exercise and Health Sciences, \\ Loughborough University, Loughborough, United Kingdom
}

\section{abstract}

Background:

Material and methods:

Results: At baseline (i.e., absence of purslane supplementation) the HIIE protocol increased 9-HODE $(p<0.001), 13-$ HODE $(p<0.001)$, IL-17 $(p<0.001)$, TNF- $\alpha(p<0.001)$, and LDH $(p<0.001)$. After purslane consumption, resting 9-HODE, 13-HODE, IL-17, TNF- $\alpha$, and LDH were lowered $(p<0.001)$ and IL-10 was increased $(p<$ 0.001 ) compared to before purslane supplementation. After purslane consumption and following the HIIE protocol, the responses of these biomarkers were lower compared to baseline $(p<0.001)$.

Conclusions:

Key words:

Ten days of purslane supplementation blunted the perturbation to biomarkers of oxidative stress, inflammation and muscle damage in female runners completing a HIIE protocol.

\section{article details}

Article statistics:

Full-text PDF:

Received: September 2020; Accepted: February 2021; Published: March 2021

Copyright

Indexation:

http://www.balticsportscience.com

(c) Gdansk University of Physical Education and Sport, Poland

Celdes, Clarivate Analytics Emerging Sources Citation Index (ESCI), CNKI Scholar (China National Knowledge Infrastructure), CNPIEC, DOAJ, EBSCO - Central \& Eastern European Academic Source, EBSCO - SPORTDiscus, EBSCO Discovery Service, Google Scholar, Index Copernicus, J-Gate, Naviga (Softweco, Primo Central (ExLibris), ProQuest - Family Health, ProQuest - Health \& Medical Complete, ProQuest - Illustrata: Health Sciences, ProQuest Nursing \& Allied Health Source, Summon (Serials Solutions/ProQuest, TDOne (TDNet), Ulrich's Periodicals Directory/ ulrichsweb, WorldCat (OCLC)

Funding: This research received no specific grant from any funding agency in the public, commercial, or not-for-profit sectors.

Conflict of interests:

Corresponding author:

Open Access License:

Amine Ghram, Department of Exercise Physiology, Faculty of Physical Education and Sport Sciences, University of Tehran, 14398-13117 Tehran, Iran ; e-mail: amineghram.110@ut.ac.ir

This is an open access article distributed under the terms of the Creative Commons Attribution-Non-Commercial-NoDerivatives 4.0 International (https://creativecommons.org/licenses/by-nc-nd/4.0/), which permits use, distribution and reproduction in any medium, provided the original work is properly cited, the use is non-commercial and is otherwise in compliance with the license. 


\section{INTRODUCTION}

The herb portulacaoleracea L. (common name purslane) has been widely used as a potherb in Central Europe, Asia, and the Mediterranean region [1]. The potential nutraceutical properties of purslane have been reported to include a wide spectrum of pharmacological properties such as neuroprotective, antimicrobial, antidiabetic, antioxidant, antiinflammatory, antiulcerogenic, and anticancer activities [2]. The intake of antioxidants could prevent exercise-induced muscle damage [3]. In addition to its potential therapeutic properties, purslane administration has been reported to interact with exercise to modulate oxidative stress responses. Specifically, purslane supplementation has been reported to lower exercise-induced oxidative stress in mice [4] in rat tissues [5], and in women with type II diabetes [6], when combined with endurance exercise training. However, the independent effects of purslane supplementation on acute exercise-induced oxidative stress in healthy female athletes have yet to be determined. One recent study showed that 10 days of purslane supplementation reduced the levels of oxidative stress (9- and 13-hydroxyoctadecadienoic acid [9-HODE, 13-HODE]), pro-inflammatory cytokines (interleukin 17 [IL-17] and tumor necrosis factor [TNF $\alpha]$ ), and muscle damage (lactate dehydrogenase [LDH]), compared to levels pre-supplementation in male runners [7]. However, studies investigating the effects of exercise on 13-HODE + 9-HODE are scarce. It was reported that 13-HODE + 9-HODE increased following a 75-km cycling bout in trained male cyclists [8] and following 2.5-km high intensity interval exercise (HIIE) on treadmill [7]. Interleukin 10 (IL-10), an immunosuppressive cytokine [9], increased substantially following endurance exercise longer than $2 \mathrm{~h}$, such as a marathon and triathlon race [10]. IL-17 tended to increase after the race in male triathletes [11] and after $2.5 \mathrm{~km}$ of HIIE bout [7]. TNF- $\alpha$ did not change significantly after a race in male triathletes [10] and increased after $2.5 \mathrm{~km}$ of HIIE bout [7]. Thus, the response of these markers is variable dependent upon the type of exercise performed.

Interval exercise can be performed in different modalities and intensities such as perceived effort, heart rate, mechanical loading and intensity associated to $\mathrm{VO}_{2} \max$ [12] and is being increasingly utilized by athletes as it may facilitate greater improvements in endurance performance compared to low-intensity continuous exercise [13]. That said, HIIE is also a potent pro-oxidant $[14,15]$ and inflammatory stimulus $[14,16]$ that provokes muscle damage [17]. The development of oxidative stress and inflammation during exercise has been implicated in the process of exercise-induced fatigue [18] and muscle damage, both of which can retard post-exercise recovery. Therefore, there is a growing interest in interventions that can mitigate exercise-induced oxidative stress, inflammation and muscle damage.

There is little information regarding the purslane supplementation on oxidative stress, inflammatory cytokines, and muscle damage after an acute bout of aerobic exercise in athletes. Therefore, the purpose of this study was to investigate the effects of an acute bout of HIIE on oxidative stress, inflammatory and muscle damage biomarkers in aerobic trained-women and whether 10-day supplementation with purslane could negate any observed differences. We hypothesized that acute HIIE results in post-exercise elevation in oxidative stress, inflammatory and muscle damage biomarkers and that the response would be lower after short-term purslane supplementation possibly because of antioxidant and anti-inflammatory activity of purslane supplementation.

\section{MATERIAL AND METHODS}

\section{SUBJECTS}

Nine national standard female middle-distance runners (age, $23 \pm 4$ yrs; body mass, 52 $\pm 3 \mathrm{~kg}$; height, $1.65 \pm 0.06 \mathrm{~m}$; and BMI, $19 \pm 1 \mathrm{~kg} / \mathrm{m}^{2}$; and peak velocity in an incremental treadmill test, $16.2 \pm 1.0 \mathrm{~km} / \mathrm{h}$ ) with normal menstrual cycles volunteered to participate 
in this study. All athletes had been competing in middle distance road races (1500 and $3000 \mathrm{~m}$ ) for at least 10 years and had competed in national competitions. They were completing a similar weekly training volume (10-14 hours) and had experience in high intensity interval training. All athletes were non-smokers, physically healthy, and had no history of health disorders. In addition, they were not taking any medications or vitamin, mineral or herbal supplements.

The study was approved by the Research Ethics Committee of the Sport Sciences Research Institute and performed in accordance with the ethical standards of the Declaration of Helsinki (IR.SSRI.REC.1397.358). The athletes were informed of the study procedure and the risks involved with the measurement. A written consent form was obtained from each athlete.

\section{EXPERIMENTAL DESIGN}

The present study was conducted over 17 days and required athletes to complete three experimental sessions during this period. After meeting the inclusion criteria, female runners performed a familiarization session at their first visit to our laboratory to ensure all they were accustomed to all of the experimental procedures. This visit also included the completion of a medical history questionnaire and the assessment of anthropometry. On this familiarization visit to the laboratory, peak velocity in an incremental treadmill test was also determined through the completion of an exhaustive incremental treadmill running test. After $72 \mathrm{~h}$ of rest, a venous blood sample was obtained before and after the HIIE test on the second visit to the laboratory. Subsequently, the athletes were required to ingest 1000 $\mathrm{mg} /$ day of purslane for a total of 10 days, with purslane consumed twice daily (500 $\mathrm{mg}$ after lunch and dinner) [7]. During these 10 days, athletes were instructed to discontinue any vitamin or antioxidant dietary supplement and were invited to follow the same dietary plan before testing sessions and during their training schedule. The day following the completion of the purslane supplementation, athletes returned to the laboratory and underwent the same assessments as described on the second laboratory visit.

\section{Purslane preparation}

The purslane supplement was prepared as previously described [5-7] with voucher specimen no.15-04979. Briefly, purslane seeds were purchased from a store in Tehran (Iran) and washed thoroughly with water and air dried at room temperature for 7 days. The dry seeds were powdered and dissolved in distilled water.

\section{ANTHROPOMETRIC MEASUREMENTS}

Height was measured to the nearest $0.1 \mathrm{~cm}$ while athletes were barefoot using a stadiometer (SECA 274, Hamburg, Germany). Body mass was measured to the nearest $0.2 \mathrm{~kg}$, with the athletes wearing light clothes and no shoes (SECA 707, Hamburg, Germany). BMI (kg/ $\mathrm{m} 2$ ) was defined as body mass ( $\mathrm{kg}$ ) divided by the square of height (m2).

\section{MaXimal enduRANCE RUNNING TEST}

The athletes completed an incremental running test on a motorized treadmill (HP Cosmos Venus, Traunstein, Germany) that was continued until volitional exhaustion, as described previously $[19,20]$. After completing an initial warm-up at5 $\mathrm{km} / \mathrm{h}$ for $3 \mathrm{~min}$, the initial speed was set at $8 \mathrm{~km} / \mathrm{h}$ and was increased by $1 \mathrm{~km} / \mathrm{h}$ after each $2 \mathrm{~min}$ stage until the athlete could no longer continue [19]. When the subject was not able to finish a 2 min stage, the peak speed was calculated by considering the percentage time completed in the final stage, as described previously [7, 19]:

Peak treadmill velocity $=$ speed of last stage complete $+[$ (time $(\mathrm{s})$ performed in the last stage/120) $\times 1$ ] 
Heart rate was monitored continuously throughout the tests with a heart rate monitor (Polar Electro FT7, Kempele, Finland). Athletes were verbally encouraged to run until exhaustion.

\section{HIGH-INTENSITY INTERMITTENT EXERCISE}

The HIIE protocol administered in the current study has been described previously [19, 21]. Briefly, the athletes performed a warm-up prior to completing the HIIE which consisted of running at $50 \%$ of peak treadmill velocity for $5 \mathrm{~min}$, at $1 \%$ inclination. Thereafter, the HIIE protocol was commenced which required subjects to complete 1 min bouts of constant velocity treadmill running at 100\% peak treadmill velocity, interspersed by 1 min of passive recovery. This protocol was continued until subjects had completed $2.5 \mathrm{~km}$ [21].

\section{BLOOd SAMPLING AND ANALYSES}

Venous blood samples were collected by a trained nurse after an overnight fast at rest and immediately after the HIIE protocol. The blood samples $(10 \mathrm{ml})$ were immediately allocated into two $5 \mathrm{~mL}$ vacutainer tubes containing EDTA for plasma separation and one $5 \mathrm{~mL}$ sterile vacutainer tube for serum separation. The tubes were centrifuged at $3500 \mathrm{rpm}$ for $15 \mathrm{~min}$ at $4^{\circ} \mathrm{C}$, and plasma and serum samples were stored at $-20^{\circ} \mathrm{C}$ until analysis. Oxidative stress markers including 9-HODE and 13-HODE were analyzed in the serum using enzyme-linked immunosorbent assay (ELISA) kits (ZellBio Gmbh, Germany and abcam, UK; respectively). Inflammatory markers including TNF- $\alpha$, IL-10, and IL-17 were analyzed by using ELISA kits (Diaclone, France). Lactate was analyzed in the serum using ELISA kit (LDH kit, Pars Azmoon, Iran).

\section{STATISTICAL ANALYSIS}

All statistical analyses and calculations were conducted using SPSS (version 21.0, Chicago, IL, USA). The Shapiro-Wilk test was conducted to verify the normality of distribution. Reliability of the dependent variables was determined by comparing physiological measurements at pre-HIIE (before purslane consumption vs. after purslane consumption) and post-HIIE (before purslane consumption vs. after purslane consumption) and was calculated using intra-class correlations (ICCs). The ICC was calculated for degree of absolute agreement and used a 2-way random-effects model. The ICC values 0.40 or less were considered poor, 0.40 to 0.59 was fair, 0.60 to 0.74 was good, 0.75 to 1.0 was excellent [22].

Two-way repeated-measures analysis of variance (ANOVA) was used to examine differences in blood measures according to trial condition (before purslane consumption vs. after purslane consumption) and time (pre-HIIE vs. post-HIIE) and interactions between these independent variables. The Tukey post hoc test was used when significant differences were revealed by the ANOVA. The magnitude of effect was described by the mean change and 95\% confidence interval (CI) of the change. Furthermore, the magnitude of the difference for each pairwise comparison was quantified using Cohen's d effect size (ES) and classified as trivial (0.0-0.2), small (0.2-0.6), moderate (0.6-1.2), large (1.2-2.0), or very large $(>2.0)$. Effect sizes were calculated using eta squared $\left(\eta^{2}\right)$. Statistical significance was set at $p<0.05$ and data are presented as a mean \pm standard deviation (SD).

\section{RESULTS}

The subjects tolerated purslane dosage well, as no side effects from the supplementation procedures were reported.

Reliability OF dePENDENT MEASURES

Reliability statistics are displayed in Table 1. The ICC values were "poor" for all dependent measures. 
Table 1. Reliability of physiological parameters

\begin{tabular}{ccc}
\hline \multirow{2}{*}{ ICC } & Pre-HIIE & Post-HIIE \\
\cline { 2 - 3 } & Before purslane vs. After purslane & Before purslane vs. After purslane \\
\hline 9-HODE $(\mathrm{ng} / \mathrm{mL})$ & 0.12 & 0.12 \\
13-HODE $(\mathrm{ng} / \mathrm{mL})$ & 0.16 & 0.16 \\
IL-10 $(\mathrm{pg} / \mathrm{mL})$ & 0.31 & 0.31 \\
IL-17 $(\mathrm{pg} / \mathrm{mL})$ & 0.04 & 0.04 \\
TNF- $\alpha(\mathrm{pg} / \mathrm{mL})$ & -0.09 & -0.09 \\
LDH $(\mathrm{mmol} / \mathrm{L})$ & -0.09 & -0.09 \\
\hline
\end{tabular}

Abbreviations: ICC, intra-class correlation; 9-HODE, 9- and 13-hydroxyoctadecadienoic acid; 13-HODE,

13-hydroxyoctadecadienoic acid; IL-17, interleukin-17; IL-10, interleukin-10; TNF- $\alpha$, tumor necrosis factor alpha; LDH, lactate dehydrogenase.

\section{Oxidative stress}

A main significant effect of condition for 9-HODE $\left(F=1025.97, p<0.001, \eta^{2}=0.99\right.$ "moderate") and 13-HODE ( $F=779.61, p<0.001, \eta^{2}=0.98$ "moderate") was found. In addition, a main significant effect of time for 9-HODE $\left(\mathrm{F}=151.47, p<0.001, \eta^{2}=\right.$ 0.94 "moderate") and 13-HODE ( $F=65.36, \mathrm{p}<0.001, \eta^{2}=0.89$ "moderate") was found. A significant condition $\times$ time interaction was found for 9-HODE $(\mathrm{F}=20.08, p=0.002$, $\eta^{2}=0.71$ "moderate") and 13-HODE ( $F=44,462, p<0.001, \eta^{2}=0.84$ "moderate").

9-HODE (Figure 1a) and 13-HODE (Figure 1b) were significantly higher post-HIIE compared with pre-HIIE before (9-HODE: mean change (95\%CI): 18.99 (14.24 to 23.73), $p<0.001$; 13-HODE: mean change (95\%CI): 9.81 (7.47 to 12.13), $p<0.001)$ and after purslane consumption (9-HODE: mean change (95\%CI): 6.56 (2.47 to 10.64), $p=0.004$; 13-HODE: mean change (95\%CI): 3.7 (2.5 to 4.89$), p<0.001)$.
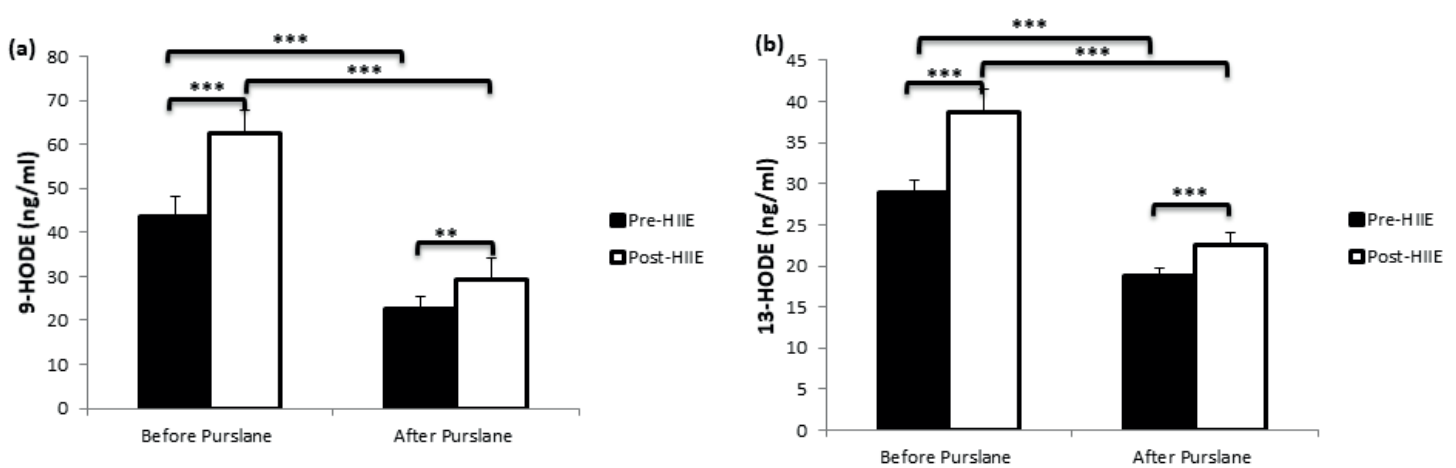

Fig. 1. Serum 9 HODE (panel a) and 13-HODE (panel b) levels pre (Pre-HIIE) and post (Post-HIIE) high-intensity intermittent exercise (HIIE) protocol before and after purslane consumption

**, *** significantly different at $\mathrm{p}<0.01 ; \mathrm{p}<0.001$, respectively.

Data are presented as means $\pm \mathrm{SD}$

9-HODE (Figure 1a) and 13-HODE (Figure 1b) were significantly lower after purslane consumption compared with before purslane consumption in pre-HIIE (9-HODE: mean change (95\%CI): -21.15 (-24.91 to -17.38), $p<0.001$; 13-HODE: mean change (95\%CI): -9.98 (11.28 to -8.67), $p<0.001$ ) and post-HIIE (9-HODE: mean change (95\%CI): -33.58 (-38.58 to -28.57), $p<0.001$; 13-HODE: mean change (95\%CI):- 16.08 (-18.36 to -13.81$), p<0.001)$. 


\section{CYTOKINES}

A main significant effect of condition for IL-10 ( $F=1160.41, p<0.001, \eta^{2}=0.99$ "moderate"), IL-17 ( $F=1769.9, p<0.001, \eta^{2}=0.99$ "moderate"), and TNF- $\alpha(F=1154.16$, $p<0.001, \eta^{2}=0.99$ "moderate") was found. In addition, a main significant effect of time for IL-10 ( $\mathrm{F}=26,970, p<0.001, \eta^{2}=0.77$ "moderate"), IL-17 ( $\mathrm{F}=125.59, p<0.001$, $\eta^{2}=0.94$ "moderate" $)$, and TNF- $\alpha\left(F=165.90, p<0.001, \eta^{2}=0.95\right.$ "moderate") was found. A significant condition $\times$ time interaction was found for IL-10 ( $F=29.84, p<0.001$, $\eta^{2}=0.78$ "moderate"), IL-17 ( $F=20.65, p<0.001, \eta^{2}=0.79$ "moderate"), and TNF- $\alpha$ $\left(\mathrm{F}=91.96, p<0.001, \eta^{2}=0.91\right.$ "moderate").
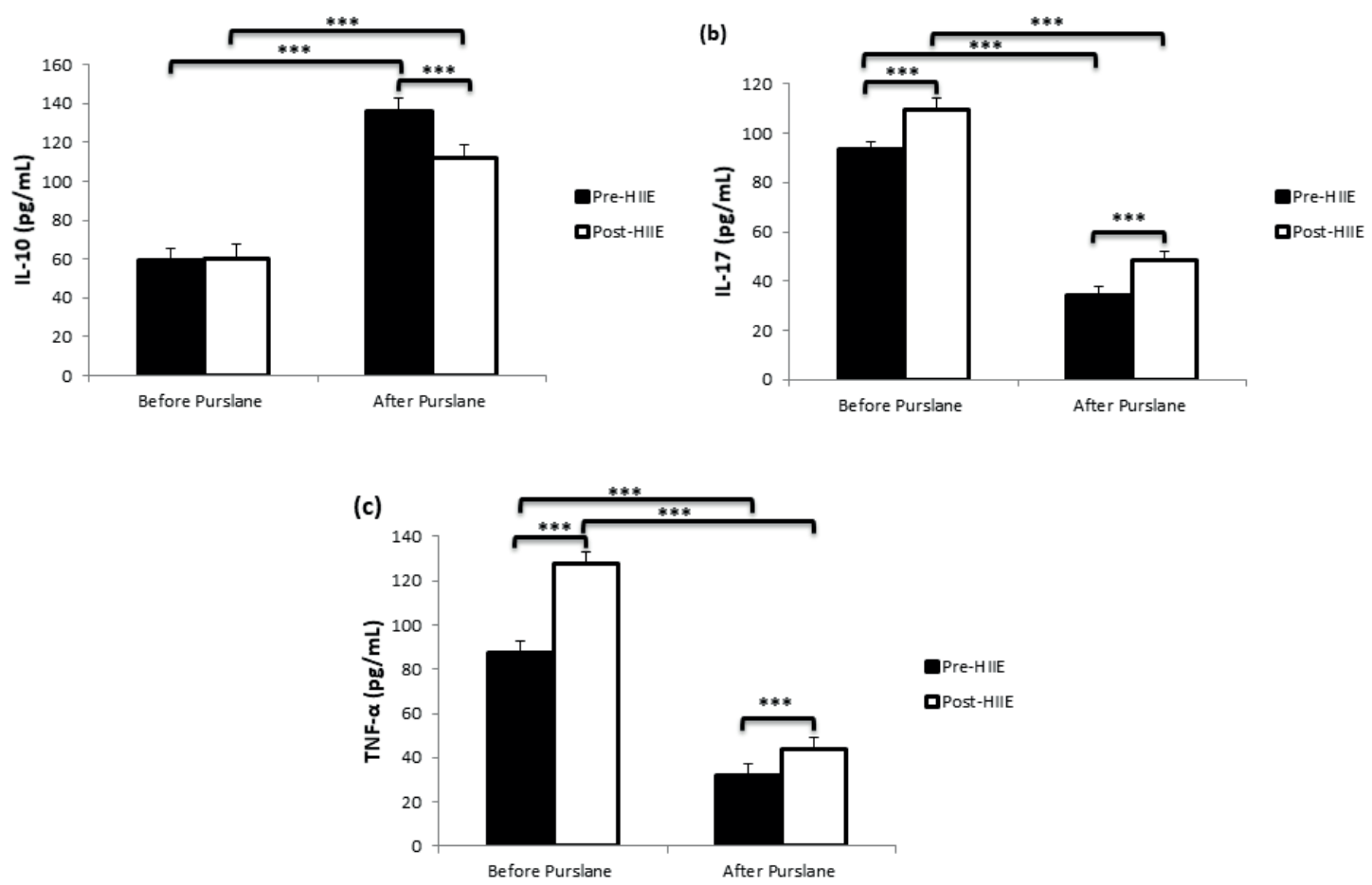

Fig. 2. Serum IL-10 (panel a), IL-17 (panel b), and TNF- $\alpha$ (panel c) levels pre (pre-HIIE) and post (post-HIIE) high-intensity intermittent exercise protocol (HIIE) before and after purslane consumption

*** significantly different at $\mathrm{p}<0.001$

Data are presented as means \pm SD

IL-10 (Figure 2a) was not significantly higher post-HIIE compared with pre-HIIE before purslane consumption (mean change (95\%CI): 1.29 (-5.54 to 8.13), $p=0.69$ ). However, IL-10 was significantly lower post-HIIE compared with pre-HIIE after purslane consumption (Il-10: mean change (95\%CI): -23.78 (-30.61 to -16.95), $p<0.001)$.

IL-17 (Figure 2b) and TNF- $\alpha$ (Figure 2c) were significantly higher post-HIIE compared with pre-HIIE before (Il-17: mean change (95\%CI): 15.94 (12.33 to 19.54), $p<0.001$; TNF- $\alpha=$ mean change (95\%CI): 39.94 (34.70 to 45.17), $p<0.001$ ) and after purslane consumption (Il-17: mean change (95\%CI): 14.2 (10.58 to 17.81), $p<0.001$ ); TNF- $\alpha$ : mean change (95\%CI): 11.87 (6.79 to 16.94), $p<0.001$ ).

IL-10 (Figure 2a) was significantly higher after purslane consumption compared with before consumption in pre-HIIE (mean change (95\%CI): 76.91 (70.27 to 83.54), $p<0.001$ ) and post-HIIE (mean change (95\%CI): 51.82 (44.79 to 58.85), $p<0.001$ ). 
IL-17 (Figure 2b) and TNF- $\alpha$ (Figure 2c) were significantly lower after purslane consumption compared with before consumption in pre-HIIE (IL-17: mean change (95\%CI): 59.65 (-62.84 to -56.45$), p<0.001$; TNF- $\alpha$ : mean change (95\%CI): -55.44 (-60.63 to -50.24$)$, $p<0.001)$ and post-HIIE (IL-17: mean change (95\%CI): -61.39 (-65.37 to -57.41 ), $p<0.001)$; TNF- $\alpha$ : mean change (95\%CI): -83.51 (-88.62 to -78.39$), p<0.001)$.

\section{MUSCLE DAMAGE}

A main significant effect of condition ( $F=1951.7, p<0.001, \eta^{2}=0.99$ "moderate") and time $\left(F=2189.67, p<0.001, \eta^{2}=0.99\right.$ "moderate") was found. A significant condition $\times$ time interaction was found for LDH concentration $\left(F=12.84, p=0.007, \eta^{2}=0.61\right.$ "moderate").

LDH (Figure 3) was significantly higher post-HIIE compared with pre-HIIE before (mean change (95\%CI): 111.44 (98.65 to 124.23$), p<0.001)$ and after purslane consumption (mean change (95\%CI): 145.77 (131.62 to 159.92), $p<0.001$ ).

LDH (Figure 3) was significantly lower after purslane consumption compared with before purslane consumption in pre-HIIE (mean change (95\%CI): -239.11 (-253.83 to -224.38), $p<0.001$ ) and post-HIIE (mean change (95\%CI): -204.77 (-216.77 to -192.78$), p<0.001$ ).

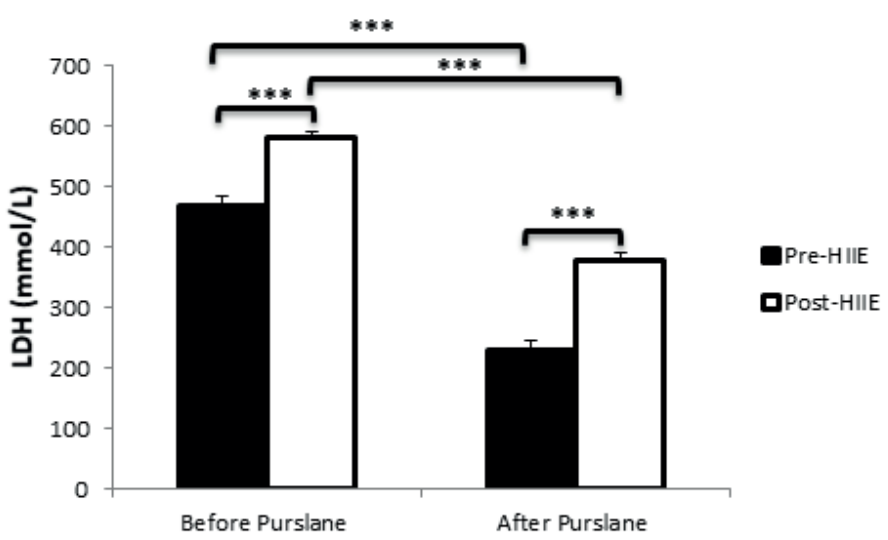

Fig. 3. Serum LDH concentration pre (pre-HIIE) and post (post-HIIE) high-intensity intermittent exercise (HIIE) protocol before and after purslane consumption

*** significantly different at $\mathrm{p}<0.001$

Data are presented as means $\pm \mathrm{SD}$.

\section{DISCUSSION}

The HIIE protocol evoked an increase in oxidative stress, inflammatory and muscle damage biomarkers consistent with previous observations. That said, 10 days of supplementation with purslane lowered oxidative stress (9-HODE and 13-HODE), pro-inflammatory (IL-17, and TNF- $\alpha$ ) and muscle damage (LDH) biomarkers before and after the HIIE protocol compared to the baseline condition without purslane supplementation. These novel observations suggest that short term supplementation with purslane can modulate oxidative stress, inflammatory, and muscle damage responses to HIIE in well-trained female runners, which might have implications for improving performance during, and expediting recovery following HIIE.

Cytokines transmit inflammatory/anti-inflammatory signals between cells and are fundamental and pleiotropic mediators of inflammation. Accordingly, cytokines are involved in regulating the pro-inflammatory response evoked by exercise-induced muscle damage [23].The production of pro- and anti-inflammatory cytokines is related the intensity and 
duration of exercise [24]. In the present study, HIIE increased the serum content of the pro-inflammatory cytokines (IL-17 and TNF- $\alpha$ ) and lowered the serum content of the antiinflammatory cytokine (IL-10). These findings are in line with previous studies. TNF- $\alpha$ increased after $5 \mathrm{~km}$ steady state exercise [19] and acute session of HIIT in recreationally active males [16]. Contradictory to our findings, TNF- $\alpha$ did not change after 24 minutes moderate-intensity continuous exercise and HIIE in healthy older adults [25] and $5 \mathrm{~km}$ HIIE in physically active male subjects [19]. IL-10 increased after $1.25 \mathrm{~km}$ HIIE, $2.5 \mathrm{~km}$ HIIE [21], and $5 \mathrm{~km} \mathrm{HIIE} \mathrm{in} \mathrm{physically} \mathrm{active} \mathrm{male} \mathrm{subjects} \mathrm{[19],} 30 \mathrm{~min}$ in both HIIE $\left(10 \times 1\right.$-min at $\left.90 \% \mathrm{VO}_{2} \max \right)$ and high intensity steady-state cycling (80\% VO2max, 20 min) in untrained males [14], acute session of high intensity interval training (HIIT) in recreationally active males [16], four bouts lower body Wingate tests in male judo athletes [26], and 24 minutes moderate-intensity continuous exercise (40\% of peak power output [PPO]) and HIIE $(12 \times 1$ min intervals at $70 \%$ PPO separated by 1 min periods at $10 \%$ PPO) in healthy older adults [25].

The complex relationship between oxidative stress and exercise is also influenced by the mode, intensity, and duration of exercise [27]. The increase of IL-17 and TNF- $\alpha$ levels can be explained by the increased glycogenolysis and lipolysis, for maintenance of the muscle contraction during exercise [26]. Future studies are needed for a better understanding of the mechanisms involved in HIIE.

In addition to modulating cytokines, the HIIE protocol increased the serum content of 9-HODE and 13-HODE in trained female runners in the present study. Moreover, elevated levels of plasma 9-HODE and 13-HODE can be reduced through the adoption of healthy diets or weight loss, and they have emerged as important indicators of oxidative stress [28]. These observations suggest that the HIIE may have induced systemic oxidative stress, which occurs when the production of oxidants outweighs the antioxidant capacity of an organism. Oxidative stress may progress to oxidative damage of cellular proteins (contractile, structural, and enzymatic), lipids, DNA, and other molecules that might conflate to impair skeletal muscle function [29]. However, more studies are necessary to better understand the involved mechanisms.

Serum LDH concentration was increased, suggestive of an increase in muscle damage, following the HIIE protocol completed in the current study. This finding is in accordance with previous studies. The lactate levels increased immediately after $5 \mathrm{~km}$ HIIE [19], four bouts of lower body HIIE Wingate test in male judo athletes [26], and sprint interval exercise in elite handball players [30, 31]. The severity of muscle damage appears to depend on multiple factors, such as the type of muscle contractions and the exercise intensity [32], with shorter duration higher intensity intervals causing greater muscle damage compared to longer duration lower intensity intervals [33], as well as the menstrual cycle [34]. Therefore, the HIIE protocol imposed in the current study was successful at increasing some pro-inflammatory, oxidative stress and muscle damage biomarkers. Future studies are needed to better understand the interplay between inflammatory, oxidative and muscle damage responses. Purslane supplementation reduced 9-HODE, 13-HODE, IL17, TNF- $\alpha$ and LDH and increased IL-10 before and after the HIIE protocol administered in the current study in female runners. Purslane supplementation has previously been reported to lower exercise-induced oxidative stress in mice completing a forced swimming test [4], in rat tissues [5], and in women with type II diabetes [6], when combined with endurance exercise training, and in male athletes runners after a HIIE bout [7]. However, our current novel observations suggest that purslane supplementation has the potential to blunt the degree of oxidative stress, inflammation and muscle damage incurred by an acute HIIE session. Purslane supplementation can also increase antioxidant status, and lower oxidative stress and inflammatory biomarkers as found in diabetic rats [35] and 
adult male Wister albino rats [36]. However, while purslane supplementation has been suggested to be an effective and safe alternative therapy for type-2 diabetic humans [37], consumption of purslane seeds for 5 weeks did not improve oxidative stress biomarkers in type 2 diabetes patients [38]. Therefore, further research is required to assess the ergogenic and therapeutic potential of purslane supplementation. In addition, these findings should be confirmed in other athletic populations.

Our study has some limitations that must be acknowledged. Since we did not fully control the athlete's diet, athletes may have consumed different foods or nutrients between the laboratory testing sessions that could have impacted the biomarkers assessed in the current study. Moreover, since we only assessed a limited number of biomarkers in the present study, further research is needed to verify these findings using a more comprehensive battery of oxidative stress, inflammatory and muscle damage biomarkers. A $1000 \mathrm{mg} /$ day of purslane seeds was administered in the current study but, since the optimal dose and duration of purslane supplementation has yet to be resolved, we cannot exclude the possibility that the effects of purslane supplementation could have been greater with a higher dose or longer duration of purslane supplementation. Finally, it is unclear whether the effects observed in the current study would have been the same after different types of exercise (different intensities and durations) or whether purslane supplementation can influence exercise performance, post-exercise recovery and the adaptations to chronic exercise training.

\section{CONCLUSION}

In summary, an acute bout of HIIE provoked increases in the systemic oxidative stress, pro-inflammatory and muscle damage markers in trained female runners. Short-term supplementation with purslane was shown to attenuate the increases in oxidative stress, pro-inflammatory cytokines and muscle damage biomarkers, and to elevate the antiinflammatory cytokine, IL-10 following the HIIE session compared to baseline. Our observations suggest that purslane supplementation can modulate oxidative stress, inflammation and muscle damage biomarkers in trained female runners, which might have implications for HIIE exercise performance and post-exercise recovery.

\section{ACKNOWLEDGEMENTS}

The authors thank the athletes and their coaches for their contribution to this project.

\section{REFERENCES}

[1] Uddin MK, Juraimi AS, Hossain MS, Nahar MAU, Ali ME, Rahman MM. Purslane weed (Portulaca oleracea): A prospective plant source of nutrition, omega-3 fatty acid, and antioxidant attributes. Sci World J. 2014;2014:951019. https://doi.org/10.1155/2014/951019

[2] Zhou Y-X, Xin H-L, Rahman K, Wang S-J, Peng C, Zhang H. Portulaca oleracea L.: A review of phytochemistry and pharmacological effects. BioMed Res Int. 2015;2015:925631. https://doi.org/10.1155/2015/925631

[3] Martinez-Ferran M, Sanchis-Gomar F, Lavie CJ, Lippi G, Pareja-Galeano H. Do Antioxidant vitamins prevent exercise-induced muscle damage? A systematic review. Antioxidants (Basel, Switzerland). 2020;9(5):372. https:// doi.org/10.3390/antiox9050372

[4] Xiang C, Zhang L, Xiaowei Z, Xiaojuan L. Polysaccharides from Portulaca oleracea L Improve Exercise Endurance and Decrease Oxidative Stress in Forced Swimming Mice. Tropical Journal of Pharmaceutical Research. 2014 13(2):229. https://doi.org/10.4314/tjpr.v13i2.10

[5] Soori R, Shahedi V, Akbarnejad A, Choobineh S. Biochemical changes in oxidative stress markers following endurance training and consumption of purslane seed in rats with hydrogen peroxide-induced toxicity. J Sport Sci Health. 2019;15:133. https://doi.org/10.1007/s11332-018-0501-y

[6] Dehghan F, Soori R, Gholami K, et al. Purslane (Portulaca oleracea) seed consumption and aerobic training improves biomarkers associated with atherosclerosis in women with type 2 diabetes (T2D). Sci Rep. 2016;6:37819. https:// doi.org/10.1038/srep37819

[7] Zare MM, Ghram A, Akbarnejad A, et al. Effect of purslane seed supplementation on inflammatory cytokines, oxidative stress and muscle damage in response to high-intensity intermittent exercise in national athlete runners. Sport Sci Health. 2019;16:47-54. https://doi.org/10.1038/srep37819 
[8] Nieman DC, Shanely RA, Luo B, Meaney MP, Dew DA, Pappan KL. Metabolomics approach to assessing plasma 13and 9-hydroxy-octadecadienoic acid and linoleic acid metabolite responses to 75-km cycling. Am J Physiol Regul Integr Compar Physiol. 2014;307(1):R68-74. https://doi.org/10.1152/ajpregu.00092.2014

[9] Suzuki K. Cytokine response to exercise and its modulation. Antioxidants (Basel). 2018;7(1):17. https://doi.org/10.3390/ antiox7010017

[10] Sugama K, Suzuki K, Yoshitani K, Shiraishi K, Kometani T. Urinary excretion of cytokines versus their plasma levels after endurance exercise. Exerc Immun Rev. 2013;19:29-48.

[11] Sugama K, Suzuki K, Yoshitani K, Shiraishi K, Kometani T. IL-17, neutrophil activation and muscle damage following endurance exercise. Exerc Immun Rev. 2012;18:116-27.

[12] Ghram A, Briki W, Mansoor H, Al-Mohannadi AS, Lavie CJ, Chamari K. Home-based exercise can be beneficial for counteracting sedentary behavior and physical inactivity during the COVID-19 pandemic in older adults. Postgrad Med. 30 Dec 2020. https://doi.org/10.1080/00325481.2020.1860394

[13] Iaia FM, Bangsbo J. Speed endurance training is a powerful stimulus for physiological adaptations and performance improvements of athletes. Scand J Med Sci Sport. 2010;20 Suppl 2:11-23. https://doi.org/10.1111/j.16000838.2010.01193.x

[14] Wadley AJ, Chen YW, Lip GY, Fisher JP, Aldred S. Low volume-high intensity interval exercise elicits antioxidant and anti-inflammatory effects in humans. J Sports Sci. 2016;34(1):1-9. https://doi.org/10.1080/02640414.2015.1035666

[15] Fisher G, Schwartz DD, Quindry J, et al. Lymphocyte enzymatic antioxidant responses to oxidative stress following high-intensity interval exercise. J Appl Physiol. (Bethesda, Md : 1985). 2011;110(3):730-7. https://doi.org/10.1152/ japplphysiol.00575.2010

[16] Zwetsloot KA, John CS, Lawrence MM, Battista RA, Shanely RA. High-intensity interval training induces a modest systemic inflammatory response in active, young men. J Inflamm Res. 2014;7:9-17. https://doi.org/10.2147/JIR.S54721

[17] Rodrigues BM, Dantas E, de Salles BF, et al. Creatine kinase and lactate dehydrogenase responses after upper-body resistance exercise with different rest intervals. J Strength Condi Res. 2010;24(6):1657-62. https://doi.org/10.1519/ JSC.0b013e3181d8e6b1

[18] Kyparos A, Salonikidis K, Nikolaidis MG, Kouretas D. Short duration exhaustive aerobic exercise induces oxidative stress: a novel play-oriented volitional fatigue test. J Sport Med Phys Fitness. 2007;47(4):483-90.

[19] Cabral-Santos C, Gerosa-Neto J, Inoue DS, et al. Similar anti-inflammatory acute responses from moderate-intensity continuous and high-intensity intermittent exercise. J Sport Sci Med. 2015;14(4):849-56.

[20] Panissa VL, Azevedo NR, Julio UF, et al. Maximum number of repetitions, total weight lifted and neuromuscular fatigue in individuals with different training backgrounds. Biol Sport. 2013;30(2):131-6. https://doi. org/10.5604/20831862.1044458

[21] Cabral-Santos C, Castrillon CI, Miranda RA, et al. Inflammatory cytokines and bdnf response to high-intensity intermittent exercise: Effect the exercise volume. Front Physiol. 2016;7:509. https://doi.org/10.3389/fphys.2016.00509

[22] Cicchetti DV. Multiple comparison methods: Establishing guidelines for their valid application in neuropsychological research. J Clin Experiment Neuropsychol. 1994;16(1):155-61. https://doi.org/10.1080/01688639408402625

[23] Ostrowski K, Rohde T, Asp S, Schjerling P, Pedersen BK. Pro- and anti-inflammatory cytokine balance in strenuous exercise in humans. J Physiol. 1999;515 ( Pt 1):287-91. https://doi.org/10.1111/j.1469-7793.1999.287ad.x

[24] Pedersen BK, Febbraio MA. Muscle as an endocrine organ: focus on muscle-derived interleukin-6. Physiol Rev. 2008;88(4):1379-406. https://doi.org/10.1152/physrev.90100.2007

[25] Windsor MT, Bailey TG, Perissiou M, et al. Cytokine responses to acute exercise in healthy older adults: The effect of cardiorespiratory fitness. Front Physiol. 2018;9:203. https://doi.org/10.3389/fphys.2018.00203

[26] Lira FS, Panissa VL, Julio UF, Franchini E. Differences in metabolic and inflammatory responses in lower and upper body high-intensity intermittent exercise. Eur J Appl Physiol. 2015;115(7):1467-74. https://doi.org/10.1007/s00421015-3127-7

[27] Bloomer RJ. Effect of exercise on oxidative stress biomarkers. Adv Clin Chem. 2008;46:1-50. https://doi.org/10.1016/ S0065-2423(08)00401-0

[28] Collino S, Montoliu I, Martin FP, et al. Metabolic signatures of extreme longevity in northern Italian centenarians reveal a complex remodeling of lipids, amino acids, and gut microbiota metabolism. PloS One. 2013;8(3):e56564. https://doi.org/10.1371/journal.pone.0056564

[29] Powers SK, Jackson MJ. Exercise-induced oxidative stress: cellular mechanisms and impact on muscle force production. Physiol Rev. 2008;88(4):1243-76. https://doi.org/10.1152/physrev.00031.2007

[30] Meckel Y, Eliakim A, Seraev M, et al. The effect of a brief sprint interval exercise on growth factors and inflammatory mediators. J Strength Cond Res. 2009;23(1):225-30. https://doi.org/10.1519/JSC.0b013e3181876a9a

[31] Meckel Y, Nemet D, Bar-Sela S, et al. Hormonal and Inflammatory responses to different types of sprint interval training. J Strength Cond Res. 2011;25(8):2161-9. https://doi.org/10.1519/JSC.0b013e3181dc4571

[32] Chen TC, Nosaka K, Sacco P. Intensity of eccentric exercise, shift of optimum angle, and the magnitude of repeatedbout effect. J Appl Physiol. (Bethesda, Md : 1985). 2007;102(3):992-9. https://doi.org/10.1152/japplphysiol.00425.2006

[33] Wiewelhove T, Fernandez-Fernandez J, Raeder C, et al. Acute responses and muscle damage in different high-intensity interval running protocols. J Sport Med Phys Fitness. 2016;56(5):606-15.

[34] Tiidus PM. Estrogen and Menopause: muscle damage, repair and function in females. In: Hackney AC, editor. Sex hormones, exercise and women: scientific and clinical aspects. Cham: Springer International Publishing; 2017; 7185. https://doi.org/10.1007/978-3-319-44558-8_5

[35] Samarghandian S, Borji A, Farkhondeh T. Attenuation of Oxidative Stress and Inflammation by Portulaca oleracea in Streptozotocin-Induced Diabetic Rats. J Evidence-Based Complement Altern Med. 2017;22(4):562-6. https://doi. $\operatorname{org} / 10.1177 / 2156587217692491$

[36] Dkhil MA, Moniem AEA, Al-Quraishy S, Saleh RA. Antioxidant effect of purslane (Portulaca oleracea) and its mechanism of action. J Medicin Plant Res. 2011;5(9):1589-93. 
Baltic Journal of Health and Physical Activity 2021; 13 (1): 17-27

Journal of Gdansk University of Physical Education and Sport

e-ISSN 2080-9999

[37] El-Sayed M-IK. Effects of Portulaca oleracea L. seeds in treatment of type-2 diabetes mellitus patients as adjunctive and alternative therapy. J Ethnopharmacol. 2011;137(1):643-51. https://doi.org/10.1016/j.jep.2011.06.020

[38] Zakizadeh E, Faghihimani E, Saneei P, Esmaillzadeh A. The effect of purslane seeds on biomarkers of oxidative stress in diabetic patients: A randomized controlled cross-over clinical trial. Int J Prev Med. 2015;6:95. https://doi. org/10.4103/2008-7802.166505

Cite this article as:

Charkhi Sahl Abad A, Ghram A, Soori R, Akbarnejad A, Azizi Ghuchan F, Zare MM, Hackney AC, Bailey S].

Purslane supplementation lowers oxidative stress, inflammatory and muscle damage biomarkers after high-intensity intermittent exercise in female runners

Balt J Health Phys Act. 2021;13(1):17-27

doi: 10.29359/BJHPA.13.1.03 